\title{
An Selection System For Automotive Sentiment Classification In Hadoop Using KNN Classifier
}

\author{
Vimal Kumar Stephen K, V Mathivanan, Anoud Saleh Rashid Al-Alawi, and Sausan Shinoon Al-Sulti
}

\begin{abstract}
With the growing popularity of big data analytics in the area of online product review, the biggest issue is voluminous data. Sentiment analysis and opinion mining are useful for solving text and web based issues. For sentiment analysis, this work makes use of the Hadoop framework. The Hadoop is not only reliable but also a fault immune model for processing huge amounts of data. There is a critical role that is played by sentiment analysis in text mining purposes such as in consumer attitude recognition, trade name and product spotting, customer relationship management, and market research. Data is labelled either as subjective or objective based on the subjectivity classification. This subjective classification is further divided as positive, negative or neutral by sentiment classification. The sentiment is classified based on the features which are taken from the data. As feature selection contributes in conserving the classification expense with regard to time and computation load, feature selection has gained a lot of prominence. This work uses the Term Frequency (TF) feature extraction. The objective here is using feature selection based on information Gain (IG) and Particle Swarm Optimization (PSO) for feature selection in sentiment classification. These schemes can decrease the features in the original set as they eliminate redundant features for text sentiment categorization and thus improvise the accuracy of classification. Also, the running time of the learning algorithms is decreased. K-nearest neighbour (KNN) classifier is used for evaluating the suggested scheme. It has been shown by empirical outcomes that compared to the IG based feature selection; the PSO based feature selection scheme attains better and more robust performance.
\end{abstract}

Index Terms - Big Data, Sentiment Analysis, Hadoop Framework, Feature Selection, Information Gain (IG), Particle Swarm Optimization (PSO), Naïve Bayes, and KNearest Neighbor (KNN).

\section{INTRODUCTION}

Several applications for handling big data are becoming pervasive. Nonetheless, it is the analysis of big data which is the biggest challenge. A framework called MapReduce has gained a lot of prominence for this kind of dataintensive application(s). Scalable parallel applications can be formulated using the MapReduce programming module; data can be processed in huge clusters of ${ }^{1}$ commercial machines. Some examples of MapReduce

Published on December 15, 2019.

Vimal Kumar Stephen K, Department of Information Technology

Ibra College of Technology, Sultante of Oman

(e-mail: vimal@ict.edu.om).

V Mathivanan, Department of Information Technology

Ibra College of Technology, Sultante of Oman.

(e-mail: vimal@ict.edu.om). frameworks are Google's MapReduce or open source equivalent of Hadoop [1].

In today's world, a huge quantity of data of the order of quintillion bytes of data are generated. Such voluminous data should be effectively and resiliently processed. There is a huge effort for coping with loads of data by the researchers from the instance perspective. However, the research community has severely disregarded the opposite side despite there being adverse implications. There are some considerable issues which need to be addressed by the feature selectors in big data, particularly the ones that are based on pair wise correlations. This is because they can shift rapidly to trillions of features when facing millions of them.

For tackling big data, there are many scalable tools and technologies that have emerged in the recent years. Transparent high-level distributed processing services are provided by most of them to the end users. The pioneering programs in these areas are MapReduce and its open source version Hadoop. Of late, there has been an emergence of second generation of tools to counter the weaknesses of first generation tools.For example; Apache spark is gradually supplanting disk intensive specialization in Hadoop. The former offers faster memory based primitives. Spark aims at speeding up interactive, online and iterative schemes that as usually existent in machine learning (ML) algorithms, according to the authors. A large number of big-scaled ML libraries are spawned by the big data frameworks. These have aided the ML algorithms to perform with huge databases without being affected. For instance, MLlib depends on Spark's operations for pacing the changes between the iterations in ML. Despite the advancement in the existing MLlib, there are several drawbacks compared to the other data pre-processing applications [2].

There are several huge datasets of computer clusters of commercial hardware along with which Hadoop is used. Apache Hadoop is the open source code framework. It is provided by java. There are several layers in Hadoop. For storage, Hadoop Distributed File System (HDFS)is used. The programming schema that is employed here is MapReduce. Additionally, other high-level programming languages are also used. A few

Anoud Saleh Rashid Al-Alawi, Department of Information

Technology, Ibra College of Technology, Sultante of Oman. (e-mail: 36S1463@ict.edu.om)

Sausan Shinoon Al-Sulti, Department of Information

Technology, Ibra College of Technology, Sultante of Oman. (e-mail: 36S1498@ict.edu.om) 
are- Hbase which is a sparse database which can store huge data volumes, pig which is the data flow oriented language that uses Pig Latin programming language and Hive - a database language by SQL-92 queries. Many huge data solutions are available in today's world and among them , Apache Hadoop is the most preferred.

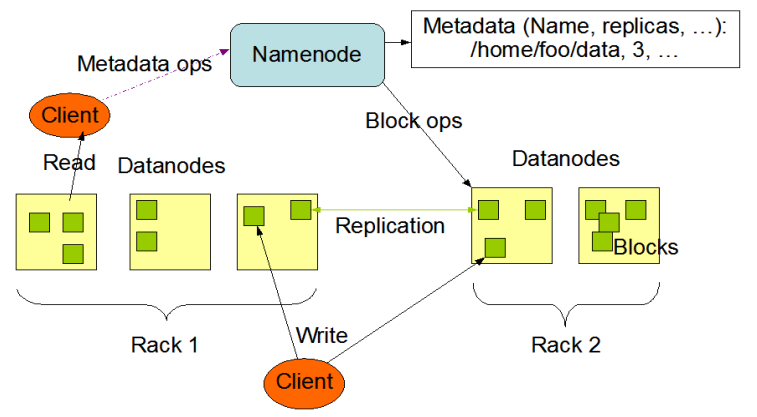

Fig. 1. Hadoop Distributed File System (HDFS) Architecture

There are several thousands of database on which Linux runs. On these, there is a master machine which is in charge of the storage tasks- "Data Nodes" and processing tasks "Task Tracker". The components managing storage tasks and processing tasks are respectively referred to as "Name Node" and "Job Tracker". Superfluous and distributed storage system, HDFS is used by the Hadoop Apache framework for storing as well as replicating file segments on several machines. The task of splitting and replicating the data in the nodes on the other workers for storage and processing is carried out by a master node. Observe fig 1.MapReduce programming model is the basis for Hadoop. There are two purposes to be printed by the user in the MapReduce: The problems are divided into smaller ones by "Map" function. The results are combined by the "Reduce function" in order that all the features of the distributed and parallel computations are abstracted [3].

The non-parametric techniques in ML literature are referred to as instance-based or memory based learning algorithms. This is because they accumulate the training cases in a lookup table and use this for interpolation. The $\mathrm{KNN}$ algorithm is the most basic instance based scheme. This work suggests IG and PSO based feature selection schemes in sentiment classification for Hadoop framework. These are the sections into which the remainder of the organization is divided- The associated works in literature have been discussed in the second section. The various techniques used in the work are described in the third section. Empirical outcomes are described in the fourth section and the conclusion of the work is presented in the fifth section.

\section{RELATED WORKS}

A new scheme for texture based text detection in videos that makes use of Apache Hadoop big data analysis has been suggested by Ayed et al., [3]. Video frames are decomposed by the suggested system into several blocks of fixed sizes. These are analyzed using Haar wavelet transformation for generating feature vectors. These are classified into text blocks and nontext blocks with a neural network classifier. This scheme can detect texts having different languages and fonts of various colors, sizes and orientations. The time for computation is reduced by half due to the MapReduce programming scheme. For eliminating the noisy regions and text like regions to be excluded, there is a need for further processing on the basis of the geometric traits of these regions.

The probability of solving a well known digital image forensics issues has been explored by Cattaneo et al., [4]. This issue is referred to as Source Camera Identification (SCI) setback for which distributed concept is proposed. The first coded implementation by this scheme could leverage the capability of the Hadoop framework to transform an independent Java application into a shared one having some intrusions on the original source code. Some wary profiling activity has been conducted. This will enable us to target some critical performance problems that are a result of the vanilla porting of the algorithm. An in-depth analysis of the resulting implementations has been done on a cluster comprising thirty three commercial PCs as well as a dataset of five thousand one hundred sixty images. It has been shown by empirical outcomes that the optimized implementations are able to scale better with rise in quantity of computer nodes. At the same time, the performance exhibited will be at least twice slower than the peak hastening that can be achieved in theory.

Hadoop network has been used for implementing a MapReduce solution for ballistic image comparison by Kocakulak \& Temizel [5]. This ballistic comparison is a high data as well as computationally complex task. Google has developed MapReduce which is a programming model for providing a scalable, flexible and QoS assured IT infrastructure, specifically for highly parallel data oriented computational jobs. It has been shown by the outcomes that not only can the performance improve significantly but also, the computing resources are effectively utilized. Additionally, the experiences in programming are shared for tuning a Hadoop cluster.

A COSMOS platform has been suggested by Conejero et al., [6]. This can bolster sentiment as well as tension analysis on Twitter data. It has also been shown the way in which Open Nebula cloud environment can be used for scaling the platform with MapReduce based analysis by making use of Hadoop. It specifically describes the types of system configurations from the perspective of performance that would be the most viable. This implies the way in which the variability in analysis performance can be decreased using the virtual machines distributed in the infrastructure. A data set comprising several million Twitter messages are demonstrated by this approach and have been analyzed using two types of cloud architecture.

A scalable user profiling solution, executed on top of Hadoop MapReduce framework has been suggested by Shmueli-Scheuer et al., [7]. The prospective comprises the framework being extended with other profile 
prototypes like the hierarchical or the semantic prototypes. Another intention is incorporating the structured data sources into the framework. This work presents the in-depth analysis of huge scale user profiling prototype established in IBM above the Apache Hadoop. The problem of extracting as well as maintaining many user profiles from a huge scale data has been addressed. Firstly, an effective user profiling framework with high user profiling with assured value has been delineated. Then, a scalable execution of the suggested framework in Apache Hadoop as well as its confronts is discussed.

\section{METHODOLOGY}

Pertinent traits are chosen by the feature selection process based on some measurement. The purpose of feature selection is simplifying the training as well as a reduction in the training duration. This work makes use of a subset of amazon automotive product review dataset (15000 positive, 20000 negative and 15000 neutral). Feature selection is done using IG based feature selection, PSO based feature selection and feature extraction is done by TF method. The feature selection techniques are evaluated by means of KNN classifier.

\section{A. Term Frequency (TF) Feature Extraction}

The frequency with which a provided term comes in a certain text or a document is known as the term frequency or TF [8]. For instance, if a document contains one hundred words and the word 'dog' comes thrice, then the TF for ' $d o g$ ' is $3 / 100=0.03$. Thus the frequency of a term $t$ (word/token) in a document $d$ is known as TF. Naturally, the occurrence of a term in a longer document may be more than that in a shorter document as every document is of a variable length .Thus, the TF is regularly divided by the document length (quantity of terms intoto in a document) as a way of normalization in (1).

$$
\text { Normalized } T F=\frac{t f(t, d)}{n_{d}}
$$

Where,

$$
\begin{aligned}
& \operatorname{tf}(t, d)=\text { Term frequency } \\
& n_{d}=\text { The total number of terms in } \\
& \text { document } \mathrm{d} .
\end{aligned}
$$

\section{B. Information Gain (IG) based Feature Selection}

In machine learning based classification, IG has been employed as a feature/term good condition. This assesses if a feature in a text document is present or not. Then computes the information acquired in bits for forecasting the class of a random text document.IG is computed by the contribution of the feature in reducing the overall entropy [9]. Entropy is referred to as the expected information required for classifying an instance/tuple for partition D or identification of a class label of an instance D and is given by (2):

$$
\operatorname{Info}(D)=-\sum_{i=1}^{m}\left(P_{i}\right) \log _{2}\left(P_{i}\right)
$$

Here, the number of classes has been denoted by $\mathrm{m}$. For binary classification, $m=2$. The probability that an arbitrary instance in partition D belongs to class $C_{i}$ is given by $P_{i}$, estimated as $\left|C_{i}, \mathrm{D}\right| /|\mathrm{D}|$ (i.e. proportion of instances of each class or category). Alog function to the base 2 justifies the fact that it encodesinformation in bits. $\mathrm{D}$ can segregate into $\mathrm{V}$ partitions set $\left\{D_{1}, D_{2}, \ldots . ., D_{v}\right\}$, If it has to partition (classify) the instance in $\mathrm{D}$ on some feature attribute $A\left\{a_{1}, \ldots, a_{v}\right\}$. For an exact classification to be measured by (3), the amount of information is represented in bits

$$
\operatorname{Info}_{A}(D)=-\sum_{j=1}^{v} \frac{\left|D_{i}\right|}{|D|} \times \operatorname{Info}\left(D_{j}\right)
$$

Where $D_{j|/| \mathrm{D} \mid}$ is the weight of the jth partition and Info $\left(D_{j}\right)$ isthe entropy of partition $D_{j}$.

Finally Information gain by partitioning on $\mathrm{A}$ is (4):

$$
\operatorname{Information} \operatorname{Gain}(A)=\operatorname{Info}(D)-\operatorname{Info}_{A}(D)
$$

The features that are ranked as per the highest IG score are selected by it. It classifies the instances using those ranked features and thus optimizes the information required for decreasing the overall entropy.

\section{Particle Swarm Optimization (PSO) based Feature Selection}

In 1995, Kennedy suggested a swarm intelligence algorithm referred to as PSO. This draws its motivation from the social behaviour of a bird flock. PSO makes use of swarm of particles for exploring the search space in parallel for solving an optimization problem. Every particle has its own velocity as well as position for moving in the search area. Throughout the course of searching they distribute their best positions with one another for guiding the swarm towards the solution which is optimal. Numeric vectors are used for representing the position and the velocity where the lengths are normally the same as the dimensions in the search area [10].

Feature selection process must be executed in classification tasks. Here, a smaller set of all features can be obtained for achieving better classification performance. Feature selection in continuous space can be performed using the PSO, Binary Particle Swarm Optimization (BPSO) is proposed for solving discrete problems using PSO where there are two variations to the continuous PSO- first representing the position of the particle. In continuous PSO, the position of particle is a real values set where as in BPSO it becomes a binary 
vector. The other difference is that the direction of the movement of particle is not represented by the velocity any more. It signifies the prospect of selecting" 1 " at a specific bit of a particle's movement.

Based on the fitness value, in the fitness proportionate selection step, the velocity of a particle is revised at every iteration. This concept of fitness proportionate selection is observant in the genetic algorithms where based on the fitness score, a solution is selected. The higher the fitness score, the greater are the chances of the solution being selected [11].The velocity and position of a particle is represented as $(5 \&$ 6):

$$
\begin{aligned}
& v_{i d}^{t+1}=\left\{\begin{array}{c}
m r, \text { if } n_{0}==0 \\
1-m r, \text { if } n_{1}==0 \\
\frac{f_{1}}{f_{1}+f_{0}}, \text { otherwise }
\end{array}\right\} \\
& x_{i d}^{t+1}=\left\{\begin{array}{c}
0, \text { rand }<v_{i d}^{t+1} \\
1, \text { otherwise }
\end{array}\right\}
\end{aligned}
$$

Where $n_{0}$ is the number of involved particles with $\mathrm{X}_{\mathrm{id}}=0$ and $\mathrm{n}_{1}$ is the number of involved particles with $\mathrm{X}_{\mathrm{id}}=$ 1. $f_{1}$ and $f_{0}$ are evaluated as: initially, the involved particles are partitioned into two sets $\mathrm{S}_{1}$ and $\mathrm{S}_{0}$ based on whether they select 0 or 1 .Then, $f_{1}$ and $f_{0}$ are computed using the mean of fitness values of particles in $\mathrm{S}_{1}$ and $\mathrm{S}_{0}$ respectively. The fitness value of the particle is given by (7 \& 8),

$$
f(x)=\sum_{1}^{n} p_{i} x_{i}-s^{*} \max p_{i}
$$

Where

$$
s=|j| \sum_{1}^{m} w_{i j} x_{i}>c_{j}
$$

Where $p_{i}$ is the value of particle $i, w_{i j}$ is the weight of the particle $i$ for swarm $j, c_{j}$ is the size of the swarm, $n$ is the number of particles and $\mathrm{m}$ is the number of constraints.

For each particle, on the basis of the crowding value of the leader, the leader is chosen using a binary tournament. It fixes the peak size of the leader set and the associated crowding values.In case the size of the leader set exceeds the peak permitted size, the best of the leaders are retained on the basis of their crowding values. Otherwise the remaining leaders are eliminated. The swarm is then divided into two components and each component has a variant scheme for mutation. Uniform mutation is associated with the first component. Non-uniform mutation is associated with the second. Uniform mutation has exploring ability and non-uniform mutation has exploitation ability. For each component, the same leader set is available. Additionally, for a particle spawned by a different component of the swarm, every particle can be utilized as the leader. This ensures that success is shared by various components in the swarm. The final outcome is a combination of varied behaviours within the same swarm.

\section{K-Nearest Neighbor (KNN) Classifier}

The KNN has been regarded as the significant ten most powerful data mining algorithms which is a part of the lazy learning family suites which do not require any explicit training phase. It is required by this technique, however, that the data instances are stored and discovering the class labels of the $\mathrm{k}$ closest cases is used for classifying unfamiliar cases. There are many distances or similarity measures that can be calculated for determining how close the two instances are. The operation is to be executed for all input instances over the entire training set. Therefore, in the context of big data, the response time may be compromised. The KNN being a non-parametric method may be used for either classification or regression tasks [12].

A case in the $\mathrm{KNN}$ is categorized by most of the nodes of its nearest neighbour when the case is allocated to the most common class among its KNN. This has been evaluated by the distance function. In case $\mathrm{k}=1$, the case will be allocated to the nearest neighbour's class. Training samples are accumulated in n-dimensional pattern space. The pattern space is searched by an unknown sample of the KNN classifier, looking for training samples in n-dimensional pattern space as well as the unknown sample of the KNN classifier looks through the pattern space for the training samples that are nearest to the unknown samples. The closeness is described based on Euclidean distance [13], where Euclidean distance between two points,

$$
\begin{gathered}
X=\left(x_{1}, x_{2}, \ldots, x_{n}\right) \text { and } Y=\left(y_{1}, y_{2}, \ldots ., y_{n}\right) \text { is (9): } \\
d(X, Y)=\sqrt{\sum_{i=1}^{n}\left(X_{i}-Y_{i}\right)^{2}}
\end{gathered}
$$

$\mathrm{X}$ and $\mathrm{Y}$ are two compared objects and $\mathrm{n}$ is their number of attributes.

KNN works as follows:

- First the parameter $\mathrm{k}$ is determined which is the number of the nearest neighbour.

- Computing the distance between the query data and training sample.

- The distance is sorted and the nearest neighbour is determined based on the kth minimal distance.

- Collecting a classification of $\mathrm{Y}$ of the nearest distance.

- For the query instance, simple majority of the classes of nearest neighbour will be the prediction value.

\section{IIII RESULTS AND DISCUSSION}

Here, the IG based feature selection, PSO based feature selection is evaluated through $\mathrm{KNN}$ classifier methods are used. The classification accuracy, average 
recall, average precision and average $\mathrm{f}$ measure as shown in tables 1 to 4 and figures 2 to 5 .

TABLE I. ClASSIFICATION ACCURACY FOR PSO BASED FEATURE SELECTION

\begin{tabular}{|c|c|c|}
\hline & $\begin{array}{c}\text { IG based feature } \\
\text { selection }\end{array}$ & $\begin{array}{c}\text { PSO based feature } \\
\text { selection }\end{array}$ \\
\hline $\mathrm{KNN}$ & 75.3 & 85.6 \\
\hline
\end{tabular}

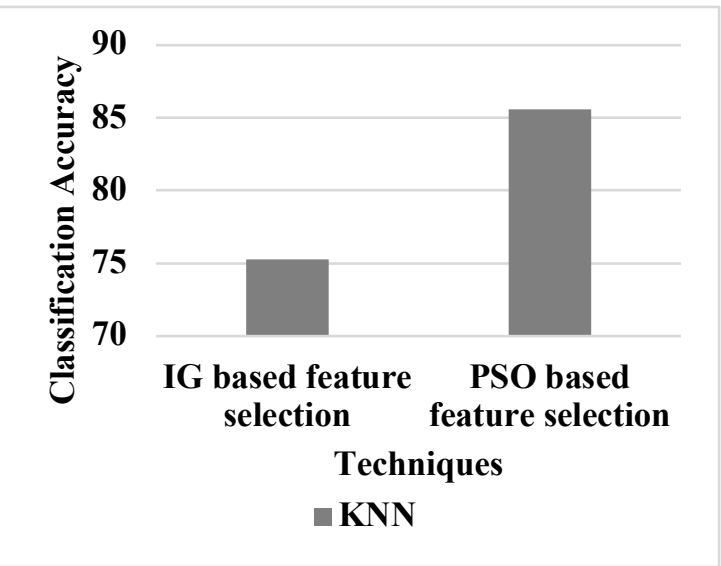

Fig. 2. Classification Accuracy for PSO based Feature Selection

From the figure 2, it can be examined that the PSO based feature selection has higher classification accuracy by $12.8 \%$ for KNN when compared with IG based feature selection.

TABLE. II. Average RECALL FOR PSO BASED FEATURE SELECTION

\begin{tabular}{|c|c|c|}
\hline & $\begin{array}{c}\text { IG based feature } \\
\text { selection }\end{array}$ & $\begin{array}{c}\text { PSO based feature } \\
\text { selection }\end{array}$ \\
\hline $\mathrm{KNN}$ & 0.754467 & 0.861667 \\
\hline
\end{tabular}

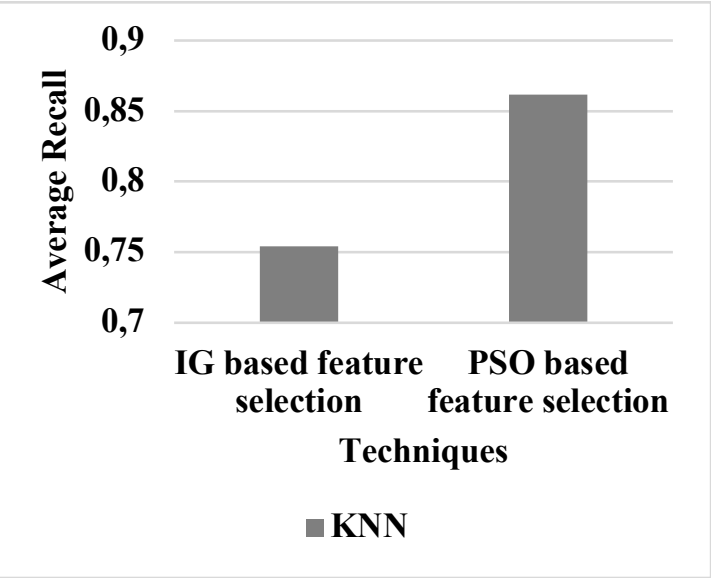

Fig. 3. Average Recall for PSO based Feature Selection

From the figure 3, it can be observed that the PSO based feature selection has higher average recall by $13.26 \%$ for KNN when compared with IG based feature selection.

TABLE III. AVERAge PRECISION FOR PSO BASED FEATURE SELECTION

\begin{tabular}{|c|c|c|}
\hline & $\begin{array}{c}\text { IG based feature } \\
\text { selection }\end{array}$ & $\begin{array}{c}\text { PSO based } \\
\text { feature selection }\end{array}$ \\
\hline
\end{tabular}

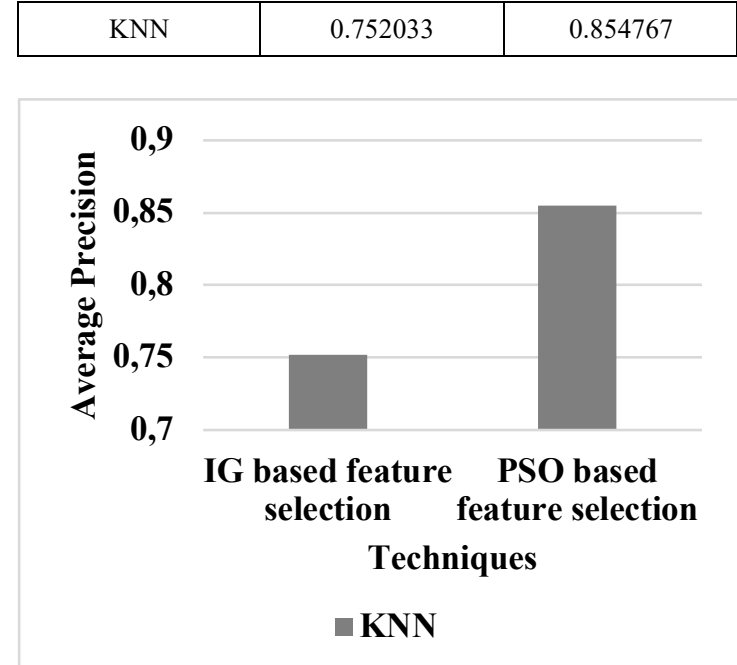

Fig. 4. Average Precision for PSO based Feature Selection

From the figure 4 , it can be observed that the PSO based feature selection has higher average precision by $12.78 \%$ for $\mathrm{KNN}$ when compared with IG based feature selection.

TABLE IIII. AVERAGE F MEASURE FOR PSO BASED FEATURE SELECTION

\begin{tabular}{|c|c|c|}
\hline & $\begin{array}{c}\text { IG based feature } \\
\text { selection }\end{array}$ & $\begin{array}{c}\text { PSO based } \\
\text { feature selection }\end{array}$ \\
\hline $\mathrm{KNN}$ & 0.7499 & 0.855367 \\
\hline
\end{tabular}

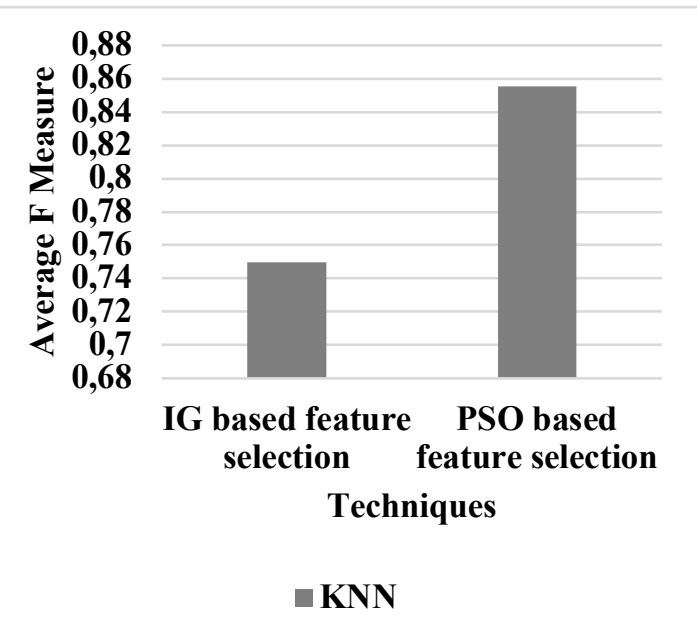

Fig. 5. Average F Measure for PSO based Feature Selection

From the figure 5, it can be observed that the PSO based feature selection has higher average $f$ measure by $13.14 \%$ for KNN when compared with IG based feature selection.

\section{CONCLUSION}

With the rapid advancement of data, it has become an utmost necessity to process this data at high speeds. Hadoop and MapReduce address this concern. One analytics scheme that conveys the thoughts of writers or producers is sentiment analysis. Thus, in big data mining environment, feature selection assumes a prominent 
role. The selection of features as well as high dimensionality is critical as various troubles are examined and estimated using different types of methodologies. This work suggests the IG and PSO based feature selection schemes. The former being a popular measure is used along with a filtering scheme for feature selection. The IG scores of all features are ranked from high to low. This work proposes a novel PSO based feature selection. Here, the PSO is guided by a newly developed fitness function for automatically selecting several original features and attaining feature selection for domain adaptation. BPSO is adapted to feature subset ranking to explore diverse feature subsets. For avoiding redundancy and noise, BPSO can effectively look for subsets of complementary features. $\mathrm{KNN}$ classifier is used. The former is easy for comprehension and implementation and also for training noisy data performing well in applications comprising a sample having many class labels. Results shows that the PSO based feature selection has greater classification accuracy by $12.8 \%$ for KNN when compared with IG based feature selection.

\section{REFERENCES}

[1] Shim, K. (2012). MapReduce algorithms for big data analysis. Proceedings of the VLDB Endowment, 5(12), 2016-2017.

[2] Ramírez-Gallego, S., García, S., Xiong, N., \& Herrera, F. (2018) BELIEF: A distance-based redundancy-proof feature selection method for Big Data. arXiv preprint arXiv:1804.05774.

[3] Ayed, A. B., Halima, M. B., \& Alimi, A. M. (2015). MapReduce Based Text Detection in Big Data Natural Scene Videos. Procedia Computer Science, 53, 216-223.

[4] Cattaneo, G., Roscigno, G., \& Petrillo, U. F. (2014, May). A scalable approach to source camera identification over Hadoop. In 2014 IEEE 28th International Conference on Advanced Information Networking and Applications (AINA) (pp. 366373). IEEE.

[5] Kocakulak, H., \& Temizel, T. T. (2011, July). A Hadoop solution for ballistic image analysis and recognition. In High Performance Computing and Simulation (HPCS), 2011 International Conference on (pp. 836-842). IEEE.

[6] Conejero, J., Burnap, P., Rana, O., \& Morgan, J. (2013, June) Scaling archived social media data analysis using a hadoop cloud. In Cloud Computing (CLOUD), 2013 IEEE Sixth International Conference on (pp. 685-692). IEEE.

[7] Shmueli-Scheuer, M., Roitman, H., Carmel, D., Mass, Y., \& Konopnicki, D. (2010, April). Extracting user profiles from large scale data. In Proceedings of the 2010 Workshop on Massive Data Analytics on the Cloud (p. 4). ACM.

[8] Kumar, J. A., \& Abirami, S. (2015). An Experimental Study Of Feature Extraction Techniques In Opinion Mining. International Journal on Soft Computing, Artificial Intelligence and Applications (IJSCAI), 4(1).

[9] Sharma, A., \& Dey, S. (2012). Performance investigation of feature selection methods and sentiment lexicons for sentiment analysis. IJCA Special Issue on Advanced Computing and Communication Technologies for HPC Applications, 3, 15-20.

[10] Nguyen, B. H., Xue, B., \& Andreae, P. (2018). A Particle Swarm Optimization based Feature Selection Approach to Transfer Learning in Classification.

[11] Suganya, B., \& Priya, V. (2017). Particle Swarm Optimization Based Feature Selection and Summarization of Customer Reviews. International Conference on Emerging trends in Engineering, Science and Sustainable Technology (ICETSST), 131-135.

[12] Maillo, J., Triguero, I., \& Herrera, F. (2015, August). A mapreduce-based k-nearest neighbor approach for big data classification. In Trustcom/BigDataSE/ISPA, 2015 IEEE (Vol. 2, pp. 167-172). IEEE.

[13] Maillo, J., Ramírez, S., Triguero, I., \& Herrera, F. (2017). kNNIS: An Iterative Spark-based design of the k-Nearest Neighbors classifier for big data. Knowledge-Based Systems, 117, 3-15.

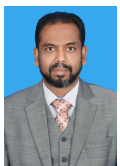

Dr. Vimal Kumar Stephen, Specilizes in Networking. He holds a CCNA, and teaches at Ibra college of technology. He published 10 international journals and 2 conferences.vimal@ict.edu.om

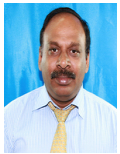

Dr. V Mathivanan, Specilizes in Networking. He holds a CCNA, and teaches at Ibra college of technology $\mathrm{He}$ has published 54 international journals and 17 conferences vmathi@ict.edu.om

Anoud Saleh Rashid Al-Alawi

Database Specilization in Ibra College of Technology. 36S1463@ict.edu.om

Sausan Shinoon Al-Sulti

Database Specilization in Ibra College of Technology. 36S1498@ict.edu.om 